\title{
Polynomial functions on subsets of non-commutative rings - a link between ringsets and null-ideal sets
}

\author{
Sophie Frisch ${ }^{1, *}$ \\ ${ }^{1}$ Institut für Analysis und Zahlentheorie, Technische Universität Graz, Kopernikusgasse 24, 8010 Graz, \\ Austria
}

\begin{abstract}
Regarding polynomial functions on a subset $S$ of a non-commutative ring $R$, that is, functions induced by polynomials in $R[x]$ (whose variable commutes with the coefficients), we show connections between, on one hand, sets $S$ such that the integer-valued polynomials on $S$ form a ring, and, on the other hand, sets $S$ such that the set of polynomials in $R[x]$ that are zero on $S$ is an ideal of $R[x]$.
\end{abstract}

2010 Mathematics Subject Classification: 13F20, 16D25, 16P10, 16 S99

Keywords and phrases: polynomial mappings, polynomial functions, integer-valued polynomials, null polynomials, null ideals, matrix algebras, triangular matrices, finite rings, non-commutative rings, ringsets

\section{Introduction}

In the theory of polynomial mappings on commutative rings, there are two notable subtopics, namely, polynomial functions on finite rings, and rings of integer-valued polynomials. Here, we are concerned with generalizations of these two topics to polynomial mappings on noncommutative rings, as proposed by Loper and Werner [8], and developed further by Werner [11-14], Peruginelli [9, 10], and the present author [3-5], among others. More particularly, we will investigate connections between null ideals of polynomials on finite non-commutative rings and integer-valued polynomials on non-commutative rings.

When we talk about polynomial functions on a non-commutative ring $R$, we mean functions induced by elements of the usual polynomial ring $R[x]$ whose indeterminate $x$ commutes with the elements of $R$. Non-commutative rings $R$ for which polynomial functions have been studied include rings of quaternions [7, 11, 14], and matrix algebras [4, 5, 12].

To begin, we introduce the two objects we want to relate, null ideals and rings of integervalued polynomials, in their original, commutative setting:

When considering polynomial functions on a finite commutative ring $R$, the first thing one likes to know is the so called null ideal $\mathrm{N}(R)$ of $R[x]$ consisting of all null-polynomials, that is, polynomials such that the function induced on $R$ by substitution of the variable is zero. The null ideal is important, because the residue classes of $R[x] \bmod \mathrm{N}(R)$ correspond to the different polynomial mappings on $R$ and hence the index $[R[x]: \mathrm{N}(R)]$ indicates the number of different polynomial mappings on $R$.

\footnotetext{
*e-mail: frisch@math.tugraz.at
} 
Regarding integer-valued polynomials, they are defined, for a domain $D$ with quotient field $K$, as those polynomials $f$ in $K[x]$ such that the polynomial function defined by $f$ on $K$ takes every element of $D$ to an element of $D[1]$.

We now generalize polynomial functions to non-commutative rings.

Let $R$ be a (possibly non-commutative) ring and

$$
f=\sum_{k} c_{k} x^{k}=\sum_{k} x^{k} c_{k} \in R[x] .
$$

Then, $f$ induces two polynomial functions on $R$, namely, the right polynomial function $f_{r}: R \rightarrow R$ and the left polynomial function $f_{l}: R \rightarrow R$, where

$$
f_{r}(s)=\sum_{k} c_{k} s^{k} \quad \text { and } \quad f_{l}(s)=\sum_{k} s^{k} c_{k} .
$$

There are other generalizations of polynomial functions to polynomials with coefficients in non-commutative rings, using polynomials whose indeterminate does not commute with the coefficients. We are not concerned with this kind of generalized polynomials here. Our topic are left and right polynomial functions defined, as above, on a non-commutative ring by polynomials in the usual polynomial ring $R[x]$ whose indeterminate $x$ commutes with the elements of $R$.

Regarding these left and right polynomial functions on a non-commutative ring $R$, we notice that they do not, in general, admit a substitution homomorphism.

It may happen, for some $s$ in $R$, and $f, g \in R[x]$ that

$$
f_{r}(s) g_{r}(s) \neq(f g)_{r}(s) \text { and also } f_{l}(s) g_{l}(s) \neq(f g)_{l}(s) .
$$

In order to generalize null-ideals to polynomials on finite non-commutative rings, we consider the sets of right and left null-polynomials, respectively, on $R$. We note that, in the absence of a substitution homomorphism, neither set is necessarily an ideal of $R[x]$.

Definition 1.1. Let $R$ a ring and $f \in R[x]$. The polynomial $f$ is called a right null-polynomial on $R$ in case $f_{r}(s)=0$ for all $s \in R$, and a left null-polynomial on $R$ in case $f_{l}(s)=0$ for all $s \in R$.

We denote the sets of right and left null polynomials on $R$, respectively, by

$$
\mathrm{N}^{r}(R)=\left\{f \in R[x] \mid \forall s \in S f_{r}(s)=0\right\} \quad \text { and } \quad \mathbf{N}^{l}(R)=\left\{f \in R[x] \mid \forall s \in S f_{l}(s)=0\right\}
$$

It is immediately clear that

Fact 1.2 (Werner [13]). For every ring $R$,

1. $\mathrm{N}^{r}(R)$ is a left ideal of $R[x]$,

2. $\mathrm{N}^{l}(R)$ is a right ideal of $R[x]$.

Proof. Indeed, if $f, g \in R[x], f=\sum_{k} a_{k} x^{k}, g=\sum_{k} b_{k} x^{k}$, and $s \in R$, then

$$
(g f)_{r}(s)=\sum_{k, l} b_{k} a_{l} s^{k+l}=\sum_{k, l} b_{k} a_{l} s^{l} s^{k}=\sum_{k} b_{k}\left(\sum_{l} a_{l} s^{l}\right) s^{k}=\sum_{k} b_{k}\left(f_{r}(s)\right) s^{k}
$$

The last expression is zero whenever $f_{r}(s)=0$, which makes $\mathrm{N}^{r}(R)$ a left ideal of $R[x]$. Similarly, by interchanging left and right, we see that $\mathrm{N}^{l}(R)$ is always a right ideal of $R[x]$. 
Whether $\mathrm{N}^{r}(R)$ is also a right ideal of $R[x]$, and thus an ideal, for any finite ring $R$, is an open question, and similarly the question whether $\mathrm{N}^{l}(R)$ is a left ideal and therefore an ideal. There are no known counterexamples.

Werner has found many sufficient conditions on $R$ for $\mathrm{N}^{r}(R)$ to be a right ideal [13], but none of them are necessary. If we take $R$ as the ring of upper triangular matrices over a commutative ring $T$, we can, by judicious choice of $T$, find examples of rings violating all of Werner's necessary conditions, which nevertheless satisfy that $\mathrm{N}^{r}(R)$ is a right ideal and $\mathrm{N}^{l}(R)$ is a left ideal of $R[x]$ [5]. Such examples can also be found among rings of integer-valued polynomials on quaternions [14].

Now, when we generalize integer-valued polynomials to polynomials with coefficients in a non-commutative ring, the usual setup (as introduced in [4]) is

Definition 1.3. Let $D$ be a domain with quotient field $K$ and $A$ a finitely generated, torsionfree $D$-algebra. Let $B=A \otimes_{D} K$. To avoid certain pathologies, we stipulate that $A \cap K=D$ when $A$ and $K$ are canonically embedded in $B$.

Then, the set of right integer-valued polynomials (with coefficients in $B$ ) on $A$ is

$$
\operatorname{Int}_{B}^{\mathrm{r}}(A)=\left\{f \in B[x] \mid \forall a \in A f_{r}(a) \in A\right\}
$$

and the set of left integer-valued polynomials (with coefficients in $B$ ) on $A$ is

$$
\operatorname{Int}_{B}{ }_{B}(A)=\left\{f \in B[x] \mid \forall a \in A f_{l}(a) \in A\right\} .
$$

We remark that it is not a priori clear that $\operatorname{Int}_{B}^{\mathrm{r}}(A)$ and $\operatorname{Int}_{B}^{1}(A)$ are rings, because, in the absence of a substitution homomorphism, closure under multiplication is not a given. Yet, there are no known counterexamples. Whether $\operatorname{Int}_{B}^{\mathrm{r}}(A)$ and $\operatorname{Int}_{B}{ }_{B}(A)$ are rings for any $D$-algebra $A$ as in Definition 1.3 remains an open question.

In some cases it is possible to describe $\operatorname{Int}_{B}{ }_{B}(A)$ and $\operatorname{Int}_{B}^{1}(A)$ via their relation to the commutative $\operatorname{ring}_{\operatorname{Int}_{K}}(A)=\operatorname{Int}_{B}^{\mathrm{r}}(A) \cap K[x]=\operatorname{Int}_{B}{ }_{B}(A) \cap K[x]$, for instance, when $A=\mathrm{M}_{n}(D)$ is the ring of $n \times n$ matrices over $D$. Here, $\operatorname{Int}^{\mathrm{l}}{ }_{\mathrm{M}_{n}(K)}\left(\mathrm{M}_{n}(D)\right)$ coincides with $\operatorname{Int}^{\mathrm{r}} \mathrm{M}_{n}(K)\left(\mathrm{M}_{n}(D)\right)$ (shown to be a ring by Werner [12]), and is canonically isomorphic to $\mathrm{M}_{n}\left(\operatorname{Int}_{K}\left(\mathrm{M}_{n}(D)\right)\right.$ ) [4]. The algebras for which $\operatorname{Int}_{B}(A) \simeq \operatorname{Int}_{K}(A) \otimes_{D} A$ thus holds have been characterized by Peruginelli and Werner [10].

For $\mathrm{T}_{n}(D)$ the ring of $n \times n$ upper triangular matrices with entries in $D, \operatorname{Int}^{\mathrm{r}} \mathrm{T}_{n}(K)\left(\mathrm{T}_{n}(D)\right)$ is isomorphic to the algebra of matrices whose entries in position $(j, k)$ are $\operatorname{in}_{\operatorname{Int}_{K}}\left(\mathrm{~T}_{n-k+1}(D)\right)$, and $\operatorname{Int}^{1}{ }_{T_{n}(K)}\left(\mathrm{T}_{n}(D)\right)$ is isomorphic to the algebra of matrices whose entries in position $(j, k)$ are from $\operatorname{Int}_{K}\left(\mathrm{~T}_{j}(D)\right)$ [5]. The commutative rings of integer-valued polynomials on upper (or lower) triangular matrices (with coefficients in $K), \operatorname{Int}_{K}\left(\mathrm{~T}_{n}(D)\right)$, are of interest in their own right [2].

Again, Werner has given different sufficient conditions on $A$ for $\operatorname{Int}^{\mathrm{r}}{ }_{B}(A)$ to be a ring, but we know that these conditions are not necessary. Taking $A$ as the ring of upper triangular matrices over a judiciously chosen domain $D$ we can find examples where $\operatorname{Int}_{B}^{\mathrm{r}}(A)$ and $\operatorname{Int}_{B}{ }_{B}(A)$ are rings, but all known sufficient conditions are violated [5]. Also, such examples can be found among rings of integer-valued polynomials over quaternion algebras [14].

\section{A connection between ringsets and null-ideal sets}

We do not know whether $\operatorname{Int}_{B}^{\mathrm{r}}(A)$ is always closed under multiplication; nor do we know whether $\mathrm{N}^{r}(R)$ is always an ideal of $R[x]$. As a way out of this quandary, we widen the scope of our investigation. Following Werner [14], we consider integer-valued polynomials on subsets of $A$. 
Here, in addition to integer-valued polynomials on subsets, we will also consider nullpolynomials on subsets, and demonstrate a connection between the two.

In what follows, we will often confine ourselves to right polynomial functions, with the understanding that everything also holds, mutatis mutandis, for left polynomial functions. In the context of right polynomial functions, $f(c)$ means $f_{r}(c)$.

Definition 2.1. Let $A$ be a $D$-algebra and everything as in Definition 1.3 and $S \subseteq A$. The set of right integer-valued polynomials on $S$ is

$$
\operatorname{Int}_{B}^{r}(S, A)=\left\{f \in B[x] \mid \forall s \in S f_{r}(s) \in A\right\} .
$$

$S$ is called a right ringset if $\operatorname{Int}_{B}(S, A)$ is closed under multiplication, and hence a ring.

Likewise, the set of left integer-valued polynomials on $S$ is

$$
\operatorname{Int}_{B}^{l}(S, A)=\left\{f \in B[x] \mid \forall s \in S f_{l}(s) \in A\right\} .
$$

$S$ is called a left ringset if $\operatorname{Int}_{B}(S, A)$ is closed under multiplication, and hence a ring.

It is easy to give examples, both of ringsets and of sets that are not ringsets: For any $D$-algebra satisfying that $\bigcap_{d \in D \backslash\{0\}} d A=(0)$, which is, for instance, the case if $A$ is a free $D$ module and $D$ is a Noetherian or Krull domain, Werner [14] showed that a singleton $\{s\} \subseteq A$ is a ringset if and only if $s$ is in the center of $A$. To see "only if," suppose that $s$ does not commute with some $t \in A$. Let $d \in D \backslash\{0\}$ such that $t s-s t \notin d A$, and let $f(x)=d^{-1}(x-s)$. Then, both $f$ and $t$ are in $\operatorname{Int}_{B}(\{s\}, A)$, but $f t$ is not. Indeed, $(f t)(x)=d^{-1}(t x-s t)$ and $(f t)(s)=d^{-1}(t s-s t) \notin A$.

Note that an arbitrary union of ringsets is always a ringset, by the fact that an intersection of rings is a ring.

Definition 2.2. Let $R$ be a ring and $S$ a subset of $R$. We denote by $N_{R}^{r}(S)$ the set of polynomials $f \in R[x]$ such that for all $s \in S, f_{r}(s)=0$. We abbreviate $N_{R}^{r}(R)$ by $\mathrm{N}^{r}(R)$.

Likewise, we denote by $N_{R}^{l}(S)$ the set of polynomials $f \in R[x]$ such that for all $s \in S$, $f_{l}(s)=0$ and abbreviate $N_{R}^{l}(R)$ by $\mathrm{N}^{l}(R)$.

Remark 2.3. Note that $N_{R}^{r}(S)$ is always a left ideal of $R[x]$. This is demonstrated, just like the fact that $\mathrm{N}^{r}(R)$ is a left ideal, by equation 1 .

The question is: for which sets $S$ is $N_{R}^{r}(S)$ a right ideal?

Likewise, $N_{R}^{l}(S)$ is always a right ideal of $R[x]$, and the question is: for which sets $S$ is $N_{R}^{l}(S)$ a left ideal?

Definition 2.4. We say that $S$ (as a subset of $R$ ) is a right null-ideal set if $N_{R}^{r}(S)$ is a right ideal of $R[x]$, and hence an ideal of $R[x]$.

We say that $S$ (as a subset of $R$ ) is a left null-ideal set if $N_{R}^{l}(S)$ is a left ideal of $R[x]$, and hence an ideal of $R[x]$.

We will now give a criterion for ringsets in terms of null-ideal sets. For this purpose, we introduce null-polynomials modulo an ideal. We will later rephrase everything using null polynomials in the strict sense.

Definition 2.5. Let $R$ be a ring and $S$ a subset of $R$ and $I$ an ideal of $R$.

A polynomial $f \in R[x]$ is called a right null polynomial modulo $I$ on $S$ if $f_{r}(s) \in I$ for every $s \in S$.

A polynomial $f \in R[x]$ is called a left null-polynomial modulo $I$ on $S$ if $f_{l}(s) \in I$ for every $s \in S$.

We denote by $N_{(R \bmod I)}^{r}(S)$ the set of right null-polynomials $\bmod I$ on $S$ and by $N_{(R \bmod I)}^{l}(S)$ the set of left null-polynomials $\bmod I$ on $S$. 
Note that $N_{(R \bmod I)}^{r}(S)$ is always a left ideal of $R[x]$ - again, this can be seen by equation 1 - and that $N_{(R \bmod I)}^{l}(S)$ is always a right ideal of $R[x]$.

Definition 2.6. We call a subset $S$ of $R$ a right null-ideal set modulo $I$ if $N_{(R \bmod I)}^{r}(S)$ is a right ideal, and hence an ideal, of $R[x]$.

We call a subset $S$ of $R$ a left null-ideal set modulo $I$ if $N_{(R \bmod I)}^{l}(S)$ is a left ideal, and hence an ideal, of $R[x]$.

For basic facts about division with remainder in rings of polynomials over noncommutative rings, we refer to Hungerford [6]. In particular, recall that a polynomial $f$ has a right factor $(x-s)$ if and only if $f_{r}(s)=0$, and that the remainder of $f$ under polynomial division by $(x-s)$ from the right is $f_{r}(s)$.

In the special case of $D=\mathbb{Z}$ and $S=A$ the following has been shown, by a different argument, by Werner [13, Thm. 2.4]

Theorem 2.7. Let $A$ be a D-algebra and everything as in Definition 1.3. Let $S \subseteq A$.

Then, $S$ is a right ringset if and only if $S$ is a right null-ideal-set modulo dA for all non-zero $d \in D$.

Similarly, $S$ is a left ringset if and only if $S$ is a left null-ideal-set modulo dA for all non-zero $d \in D$.

Proof. We show the statement for right polynomial mappings. We write $f(c)$ for $f_{r}(c)$ in this context. (The left case is similar.)

Suppose $S$ is a right null-ideal-set modulo $d A$ for all non-zero $d \in D$. Let $F, G \in$ $\operatorname{Int}_{B}^{\mathrm{r}}(S, A)$. To show: $(F G) \in \operatorname{Int}_{B}^{\mathrm{r}}(S, A)$.

We write $F$ and $G$ as $F=f / d, G=g / c$, such that $f, g \in A[x], c, d \in D \backslash\{0\}$. For every $s \in S, f(s) \in d A$, and $g(s) \in c A$. Note, in particular, that $f$ is a right null-polynomial modulo $d A$ on $S$. Having represented $F G$ as $F G=(f g) /(d c)$, we need to show, for an arbitrary $s \in S$, that $(f g)(s) \in d c A$.

By division with remainder in $A[x]$ by $(x-s)$ from the right, we get

$$
g(x)=q(x)(x-s)+g(s)
$$

for some $q \in A[x]$. We know that $g(s)=c a=a c$ for some $a \in A$. For this $a \in A$,

$$
(f g)(x)=f(x) q(x)(x-s)+f(x) a c .
$$

We set $h(x)=f(x) a$.

$f$ being a right null-polynomial modulo $d A$ on $S$ implies, by the fact that $S$ is a right null-ideal-set modulo $d A$, that $h=f a$ is also a right null-polynomial modulo $d A$ on $S$, and that, therefore, $h(s) \in d A$. Finally, we see that $(f g)(s)=h(s) c$ is in $d c A$, as required.

For the reverse implication, suppose that $S$ is not a right null-ideal-set modulo $d A$ for some fixed $d \in D \backslash\{0\}$. To show: $S$ is not a right ringset.

Let $f, g$ in $A[x]$ such that $f$ is a right null-polynomial modulo $d A$ on $S$, but $(f g)$ is not. Now consider $F=d^{-1} f \in B[x]$ and $G=g \in A[x]$. Both $F$ and $G$ are $\operatorname{in}^{\operatorname{Int}^{\mathrm{r}}}{ }_{B}(S, A)$, but their product $F G=d^{-1} f g$ is not.

We now rephrase the link between ring sets and null ideal sets using null polynomials in the strict sense. Let $S \subseteq R$ and $I$ an ideal of $R$. We denote by $S+I$ the set of residue classes of elements of $S$ in $A / I$, that is, $S+I:=\{s+I \mid s \in S\}$.

Let $f \in R[x]$, and $\bar{f}$ image of $f$ in $(R / I)[x]$ under canonical projection. Then $f$ is a right null polynomial modulo $I$ on $S$ if and only if $\bar{f} \in N_{(A / I)}(S+I)$. In other words,

$$
N_{(R \bmod I)}^{r}(S)=\pi^{-1}\left(N_{(A / I)}(S+I)\right),
$$


where $\pi: R[x] \rightarrow(R / I)[x]$ is the canonical projection.

This shows that $S$ is a right null-ideal set modulo $I$ if and only if $S+I=\{s+I \mid s \in S\}$, as a subset of $R / I$, is a right null ideal set (and similarly with right replaced by left).

Theorem 2.8 (Version of Thm 2.7). Let A be a D-algebra and everything as in Definition 1.3. Let $S \subseteq A$. Then, $S$ is a right ringset if and only if, for all non-zero $d \in D, S+d A$ as a subset of $A / d A$ is a right null-ideal-set.

\section{A common framework for ringsets and null-ideal sets}

We state most facts of this section only for right polynomial functions, with the understanding that everything also holds when left is interchanged with right throughout. From now on, $f(c)$ abbreviates $f_{r}(c)$, the result of substituting $c$ for $x$ in $f$ to the right of the coefficients.

Among the known sufficient conditions on $A$ and $R$, respectively, for $\operatorname{Int}_{B}^{r}(A)$ to be a ring, and for $\mathrm{N}^{r}(R)$ to be an ideal of $R[x]$, there are identical properties that have been shown, independently, to be sufficient conditions for both questions.

We will now sketch a common generalization for $\operatorname{Int}_{B}^{r}(A)$ and $\mathrm{N}^{r}(R)$ that allows a unified treatment of both objects.

Definition 3.1. Let $R$ be a ring, $T$ a subring of $R$, and $I$ an ideal of $T$. We denote by $\operatorname{pol}_{R}^{r}(T, I)$ the set of polynomials in $R[x]$ that map every element of $T$ to an element of $I$, under right substitution.

$$
\operatorname{pol}_{R}^{r}(T, I)=\left\{f \in R[x] \mid \forall t \in T f_{r}(t) \in I\right\}
$$

More generally, let $S$ be a subset of $T$. Then we define

$$
\operatorname{pol}_{R}^{r}(S, I)=\left\{f \in R[x] \mid \forall s \in S f_{r}(s) \in I\right\}
$$

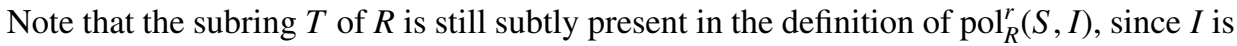
assumed to be an ideal of $T$.

In the special case where $R=T$ and $I=(0)$, we get $\operatorname{pol}_{R}^{r}(R,(0))=\mathrm{N}^{r}(R)$, the set of right null-polynomials on $R$.

When $R=T, S \subseteq T$ and $I=(0)$, we get $\operatorname{pol}_{R}^{r}(S,(0))=\mathrm{N}_{R}^{r}(S)$, the set of right nullpolynomials on a subset $S$ of $R$.

When $A$ is a $D$-algebra and $B=A \otimes_{D} K$, as in Definition 1.3, and we set $R=B$ and $T=I=A$, we have $\operatorname{pol}_{B}^{r}(A, A)=\operatorname{Int}_{B}^{\mathrm{r}}(A)$, the set of right integer-valued polynomials on $A$.

Likewise, to recover integer-valued polynomials on subsets, we set $R=B$ and $T=I=A$, and let $S$ be a subset of $A$. Then, $\operatorname{pol}_{B}^{r}(S, A)=\operatorname{Int}_{B}^{\mathrm{r}}(S, A)$ is the set of right integer-valued polynomials on $S$.

We now give an example of how integer-valued polynomials and null polynomials can be treated together in a more general setting.

Theorem 3.2. Let $C \subseteq R[x]$ and $S \subseteq T$. Then, for $\operatorname{pol}_{R}^{r}(S, I)$ to be closed under right multiplication by elements of $C$, it is sufficient that it is closed under right multiplication by the images $c_{r}(s)$ with $c \in C$ and $s \in S$.

Proof. Assume $\operatorname{pol}_{R}^{r}(S, I)$ is closed under right multiplication by elements of the form $c(s)$ with $c \in C$ and $s \in S$. Let $f \in \operatorname{pol}_{R}^{r}(S, I)$ and $c \in C$. For an arbitrary $s \in S$, we have to show that $(f c)(s) \in I$.

By division with remainder in $R[x]$ of $c$ by $(x-s)$ from the right, we get

$$
c(x)=q(x)(x-s)+c(s)
$$


for some $q \in R[x]$. Now

$$
(f c)(x)=f(x) q(x)(x-s)+f(x) c(s) .
$$

Let $h(x)=f(x) c(s)$. Then $(f c)(s)=h(s)$. By assumption, $h \in \operatorname{pol}_{R}^{r}(S, I)$, and, therefore, $(f c)(s) \in I$.

Corollary 3.3 (Werner [14, Prop. 6.13]). $\operatorname{Int}_{B}{ }_{B}(S, A)$ is a ring if and only if $\operatorname{Int}_{B}{ }_{B}(S, A)$ is closed under right multiplication by elements of $A$.

Corollary 3.4 (Werner [13, Lemma 2.3]). $\mathrm{N}_{R}^{r}(S)$ is an ideal if and only if it is closed under right multiplication by elements of $R$.

By the above corollaries, the two questions,

1. whether $\operatorname{Int}_{B}^{\mathrm{r}}(A)$ is a ring, and

2. whether $\mathrm{N}_{R}^{r}(R)$ is an ideal of $R[x]$,

can now be subsumed under a single question

3. is $\operatorname{pol}_{R}^{r}(T, I)$ a right $T$-module (with the restricion of the multiplication of $R[x]$ as scalar multiplication)?

We illustrate the principle of treating null-ideals and rings of integer-valued polynomials in one common setting by another one of Werner's sufficient conditions.

Theorem 3.5. Let $R$ be a ring, $T$ a subring of $R$ and $I$ an ideal of $T$. If $T$ is generated by units as an algebra over its center, then

1. $\operatorname{pol}_{R}^{r}(T, I)$ is a right $T$-module.

2. More generally, for every subset $S$ of $T$ that is closed under conjugation by units of $T$, $\operatorname{pol}_{R}^{r}(S, I)$ is a right $T$-module.

Proof. Let $f=\sum_{k} c_{k} x^{k} \in \operatorname{pol}_{R}^{r}(T, I)$, and $u$ a unit of $T$. Then $f u \in \operatorname{pol}_{R}^{r}(S, I)$, because, for any $t \in S, t$ can be written as $t=u^{-1} \tau u$ with $\tau=u t u^{-1} \in S$, and then

$$
(f u)(t)=\sum_{k} c_{k} u t^{k}=\sum_{k} c_{k} u u^{-1} \tau^{k} u=f(\tau) u
$$

where $f(\tau) u \in I$, because $f(\tau) \in I$ and $I$ is an ideal of $T$.

Therefore, $\operatorname{pol}_{R}^{r}(S, I)$ is closed under multiplication from the right by units of $T$. Also, $\operatorname{pol}_{R}^{r}(S, I)$ is certainly closed under multiplication from the right by elements in the center of $T$ (thanks to the fact that $S$ is a subset of $T$ ), and closed under addition and subtraction. Since every element of $T$ is a finite sum of products of central elements and units of $T$, we may conclude that $\operatorname{pol}_{R}^{r}(T, I)$ is closed under multiplication from the right by elements of $T$.

Corollary 3.6 (Werner [14, Prop. 6.13]). If A is generated by units as an algebra over its center and $S \subseteq A$ is closed under conjugation by units of $A$, then $\operatorname{Int}_{B}^{\mathrm{r}}(S, A)$ is a ring, i.e., $S$ is a right ringset.

Corollary 3.7. If $R$ is generated by units as an algebra over its center and $S \subseteq R$ is closed under conjugation by units of $R$, then $\mathrm{N}_{R}^{r}(S)$ is an ideal of $R[x]$, i.e., $S$ is a right null-ideal set.

Acknowledgement. S. Frisch is supported by the Austrian Science Fund (FWF): P 27816-N26. 


\section{References}

[1] P.-J. Cahen and J.-L. Chabert, Integer-valued polynomials, vol. 48 of Mathematical Surveys and Monographs, Amer. Math. Soc., 1997.

[2] S. Evrard, Y. Fares, and K. Johnson, Integer valued polynomials on lower triangular integer matrices, Monatsh. Math. 170, 147-160 (2013).

[3] S. FRIScH, Integer-valued polynomials on algebras - a survey, Actes des rencontres du CIRM (electronic) 2, 27-32 (2010).

[4] S. Frisch, Integer-valued polynomials on algebras, J. Algebra 373 (2013), 414-425, see also the corrigendum 412, p282 (2014).

[5] S. Frisch, Polynomial functions on upper triangular matrix algebras, Monatsh. Math. 184, 201-215 (2017).

[6] T. W. Hungerford, Algebra, vol. 73 of Graduate Texts in Mathematics, Springer-Verlag, New York-Berlin, 1980. Reprint of the 1974 original.

[7] K. Johnson and M. Pavlovski, Integer-valued polynomials on the Hurwitz ring of integral quaternions, Comm. Algebra 40, 4171-4176 (2012).

[8] K. A. Loper AND N. J. WeRner, Generalized rings of integer-valued polynomials, J. Number Theory 132, 2481-2490 (2012).

[9] G. Peruginelli and N. J. Werner, Non-triviality conditions for integer-valued polynomial rings on algebras, Monatsh. Math. 183, 177-189 (2017).

[10] G. Peruginelli and N. J. Werner, Decomposition of integer-valued polynomial algebras, J. Pure Appl. Algebra 222, 2562-2579 (2018).

[11] N. J. WERnER, Integer-valued polynomials over quaternion rings, J. Algebra 324, 17541769 (2010).

[12] N. J. WeRnER, Integer-valued polynomials over matrix rings, Comm. Algebra 40, 4717 -4726 (2012).

[13] N. J. Werner, Polynomials that kill each element of a finite ring, J. Algebra Appl. 13, 1350111, 12 (2014).

[14] N. J. WERnER, Integer-valued polynomials on algebras: a survey of recent results and open questions, in Rings, polynomials, and modules, Springer, Cham, 353-375, 2017. 